\title{
ADDITIONAL NOTES ON THE GENUS LIPPIA. XV
}

Harold N. Moldenke

LIPPIA HOEHNEI Noldenke 1978 .

Additional bibliography: Moldenke, Phytologia 39: 96--97.

Goodland describes this plant as a shrub, 1.5 meters tall, and found it growing on roadsides, flowering in July.

Additional citations: BRAZIL: Mato Grosso: Goodland 51] (N).

IIPPIA LACUNOSA Mart. \& Schau.

Additional bibliography: Hocking, Excerpt. Bot. A.25: 378 \& 379. 1975; Moldenke, Phytologia 39: 162--163 \& 453. 1978.

Burle-Marx describes this plant as a shrub, l meter tall, the corollas "pink", and found it in flower in June.

Additional citations: CULTIVATED: Brazil: Burle-Marx s.n. [Herb. Brad. 67354] (Ld).

LIPPIA LASIOCALYCINA Cham.

Additional bibliography: Moldenke, Phytologia 39: 163--164 \& 394. 1978.

Goodland reports encountering this plant in cerrado.

Additional citations: BRAZIL: Minas Gerais: Goodland $848(\mathrm{~N})$.

\section{LIPPIA LUPULINA Cham.}

Additional bibliography: Moldenke, Phytologia 39: 437.1978.

Recent collectors describe this species as an erect shrub or subshrub, $70 \mathrm{~cm}$. to $1 \mathrm{~m}$. tall, the bracts purple or "pale-purple at apex, white below", and have found it growing in "rocky terrain beside waterfalls", in "white sand on campina", and in cerrado, at $300-530 \mathrm{~m}$. altitude, flowering in November. The corollas are said to have been "dark-rose" on Hatschbach 40086, while on Prance \& al. P.24796 \& $P .25103$ they are said to have had the "corolla-tube purple, with a yellow center".

Additional citations: BRAZIL: Goiás: Hatschbach 40086 (Ld). Minas Gerais: Goodland 998 (N); Irwin, Fonsêca, Souza, Reis dos Santos, \& Ramos 27228 (Ac). Pară: Prance, Silva, Berg, Henderson, Nelson, Balick, Bahia, \& Reis dos Santos P.24796 (N), P.25103 (N). Paraná: Hatschbach 2568 (N).

LIPPIA MICROCEPHAIA Cham.

Additional bibliography: Moldenke, Phytologia 39: 437. 1978. Recent collectors describe this plant as a branched shrub, 1.2 m. tall, and have found it growing on rocky cliffs, flowering in Jamuary. The corollas are said to have been "rose" when fresh on Hatschbach 40924 .

Additional citations: BRAZIL: Minas Gerais: Hatschbach 40924 (Ld). 
IIPPIA MTCROMERA Schau.

Additional bibliography: L6pez-Palacios, Fl. Venez. Verb. 477419, 432--438, 649, \& 651, fig. 101 \& 102. 1977; Moldenke, Phytologia 39: 437 (1978) and 40: 71 \& 72.1978.

Additional illustrations: Ibpez-Palacios, Fl. Venez. Verb. [433] \& [434], fig. 101 \& 102. 1977.

Iopez-Palacios (1977) cites the following collections from Venezuela: Aragua: Trujillo 3080; Vogl 1348. Bolf́var: Bailey \& Bailey s.n.; Lasser 769; Moritz 492. Delta Amacuro: Ruiz-Terán \& Lopez-Palacios 9691. Federal District: Killip 37142; Lasser 3545 ; Vog1 127. Lara: Pittier 13108; Saer 484; Steyermark 55199; Steyermark \& Carreno 107707; Tamayo 1101, 3351; Trujillo 2550,4578 , 6546. Merida: Lobez-Palacios 1356, 2724; Lbpez-Palacios \& Bautista 3550; Ruiz-Terán \& Lbpez-Figueiras 1797, 8796; Trujil1o 6367 . Miranda: Trujillo \& 27.234. Monagas: Tamayo 3486. Sucre: Steyermark \& al. 108065, $108152,108190,108290,108315,108704 ;$ A. M. Torres 1957. Táchira: Aristeguieta 2177; Ijjasz 246; LbpezPalacios 2778; Seelkopf s.n.; Tamayo 3700; Vareschi 3999 . Trujillo: Ruiz-Terán \& López-Palacios 7592 . Margarita Island: Foldats 2658; Gines 2790, 3072, 3387, 3717; Linares 429; Preau 116; Ramia 1843: Trujillo 4917. He notes that Steyermark \& al. 108290 and 108315 were actually collected on Los Venados Island.

\section{LIPPIA MICROPHYLIA Cham.} 1978.

Additional bibliography: Moldenke, Phytologia 39: 178-179.

Recent collectors describe this species as an herb, undershrub, or slightly branched shrub, $0.8-1 \mathrm{~m}$. tall, with pale-green inflorescences, and have encountered it in dry sandy soil of savannas, rocky "campo cerrado", and "occasional" on mountain slopes, at 110 meters altitude, flowering in July and October. The corollas are said to have been "white" on Coradin \& Cordeiro 560 and "whitish" on Hatschbach 40090.

Additional citations: BRAZIL: Goiás: Hatschbach 40090 (Ld). Roraima: Coradin \& Cordeiro 560 (N), 752 (N).

\section{LIPPIA MULTIFLORA Moldenke}

Additional bibliography: Lewis \& Elvin-Lewis, Med. Bot. 391. 1977; Moldenke, Phytologia 39: 180--181 \& 445. 1978.

Lewis \& Elvin-Lewis (1977) report that the leaves of this species are used to make a native tea in western Africa.

\section{IIPPIA MYRIOCEPHAIA Schlecht. \& Cham.}

Additional bibliography: Sampson, An. Esc. Nac. Cienc. Biol. Méx. 3: 443. 1944; Hocking, Excerpt. Bot. A.25: 378. 1975; Moldenke, Phytologia 39: 438 (1978) and 40: 82. 1978; Mound \& Halsey, Whitefly World 238. 1978. 
Sampson (1944) and Mound \& Halsey (1978) list this species as host to the whitefly, Ceraleurodicus altissimus (Quaint.) Mound \& Halsey .

The Henrickson 13167, distributed as I. myriocephala, actually is L. curtisiana Moldenke, while Norris 17377, Ortega \& al. 24 , and M. Vazquez 2158 are $\mathrm{L}_{\text {. myriocephala }}$ var. hypoleia (Briq.) Koldenke.

IIPPIA MYRIOCEPHAIA var. HYPOLEIA (Briq.) Moldenke

Additional bibliography: Hocking, Excerpt. Bot. A.25: 378 . 1975; Moldenke, Phytologia 39: $438 \& 442$ (1978) and 40: 82. 1978.

Recent collectors describe this plant as a "regular" shrub or fast-growing tree, 2--8 m. tall, the fruit green or greenish, and have found it growing in alder forests, low secondary deciduous forests, and secondary oak deciduous forests, on "suelo rojizo arcilloso" or "suelo negro arcilloso, rocas calizas aflorentes, cercana a las margen de arroyos", at 1000 meters altitude, flowering in September and November. The corollas are said to have been "white" on Norris 17377 and "yellow" on M. Vazquez 2158. The latter collector reports the plant being used in rural construction work.

Additional citations: MEXICO: Hidalgo: Norris 17377 (N). Veracruz: Ortega \& al. 24 (N); M. Vazquez $\underline{2158(N)}$.

LIPPIA ORIGANOIDES H.B.K.

Additional bibliography: Garcla Barriga, Fl. Med. Colomb. 2: 508. 1975; Lobez-Palacios, Fl. Venez. Verb. 417, 418, 437--444, 450,650 , \& 651, fig. 103. 1977; Moldenke, Phytologia 39: 261-264 (1978) and 40:63. 1978.

Additional illustrations: L6pez-Palacios, Fl. Venez. Verb. [439], fig. 103. 1977.

According to Lopez-Palacios, all the Venezuelan material hitherto regarded by me as L. schomburgkiana Schau. actually represents L. origanoides growing in a xerophytic environment. He and Ruiz-Terán describe these plants as "Sufrutices erectos, hasta de $1,5 \mathrm{~m}$., con olor a espliego (no a orégano!), con deje algo desagradable, cuya epidermis caulinar se desprende en tiras largas y delgadas; capitulos axilares, en grupos de 1- 4 ; corola blanca, con garganta amarilla" and encountered it at $1450 \mathrm{~m}$. altitude, flowering in July. I am not at all convinced that these specimens are not L. schomburgkiana, but defer to Lbpez-Palacio's extensive observations and field experience. In a personal communication to me he lists the following vernacular names for I. origanoides: "orégano", "orkgano ancho", "orégano cimarrón", "orégano de cerro", "orégano de burro", "oréganon", and "poleo".

In his 1977 work, Lopez-Palacios lists the following collections from Venezuela: Aragua: Aristeguieta \& Salvidia 533; Fendler 2051. Bolivar: Curran \& Haman 199; Killip 37233; Maguire 32657; Maguire, Wurdack, \& Bunting 35868, 35970, 35997, 36021; Ruiz-Terán, 
Carabot, \& Morales 10800; Ruiz-Terán \& L6pez-Palacios 11608; Sprague s.n.; Steyermark 57640, 58601; Tamayo 3122, 3474; Trujillo $5960 ;$ Wurdack 34376; Wurdack \& Guppy 23; Wurdack \& Monachino 40974. Carabobo: Curran \& Haman 1150, 1150a; Trejo 6. Falcón: Curran \& Haman 606; Lasser \& Foldats 2956; Lasser \& Vareschi 2779; RuizTerán \& Lopez-Palacios 10287; Tamayo 975, 976 . Federal District: André $150 ;$ Birschel s.n.; Boldingh 3947; curran \& Haman 966; Debeaux 87; 0tto 456; Pittier 8096, 9671, 12725; Robinson \& Lyon s.n.; Rose \& Rose 21723a; Tamayo 4624. Guárico: Tamayo 4147. Lara: Alston 6378 ; Ferrari \& al. 322; Narcuzzi s.n.; Saer 62; Tamayo $32 \overline{26}$, $\frac{2649}{3750 ;}$ Trujil1o 6493, 6587, 6769. Mérida: Breteler 3205; I6pez-Palacios 2114, 2547; Oberwinkler 14148; Ruiz-Terán \& L6pezFigueiras 1817; Ruiz-Terán \& López-Palacios 6151; Trujillo 8007, 8106. Miranda: Pittier 8251. Sucre: Humboldt \& Bonpland 174l; Mocquerys 722; steyermark \& al. $107926,108064,108078,10 \overline{8103}$, 108193; Torres 1917. Táchira: L bpez-Palacios 2864; Tillet 737-190. Zulia: Pittier $10542 ;$ Plé 2. Margarita Island: Ginés 2768, 3069; Ramia 1851. He has also kindly pointed out that the Lasser \& Vareschi $\frac{1779}{27}$, cited by me from Bolívar in Phytologia 12: $334(\overline{1} 965)$, actually was collected in Falcón instead.

Additional \& emended citations: VENEZUELA: Bolfvar: Irwin 402 (W--2197626); Ruiz-Terán \& López-Palacios 11415 (Ld). Falcón: Lasser \& Vareschi $2779(\mathrm{Ve})$.

LIPPIA OXYCNEMIS Schau.

Additional bibliography: López-Palacios, Fl. Venez. Verb. 400 \& 651. 1977; Moldenke, Phytologia 39: 264--265. 1978.

LIPPIA OXYPHYLLARIA (Donn. Sm.) Standl.

Additional bibliography: Moldenke, Phytologia 39: 265-266. 1978.

Wilbur refers to this plant as a shrub, $2.5 \mathrm{~m}$. tall, and encountered it at 6000 feet altitude, flowering in February. The corollas are said to have been "yellow" on his no. 24313 .

Additional citations: PANAMA: Chiriqus: Wilbur $24313(\mathrm{~N})$.

LIPPIA PALMERI S. Wats.

Additional bibliography: Moldenke, Phytologia 39: 391-392. 1978 .

Additional citations: MEXICO: Sonora: W. D. Stevens 1519 (Au).

IIPPIA PENDULA Rusby

Additional bibliography: Moldenke, Phytologia 39: 393. 1978.

Boeke refers to this plant as a shrub, $2 \mathrm{~m}$. tall, "aromatic like cloves", and encountered it at 1500 meters altitude, flowerlike cloves", and encountered in April, the corollas said to have been "white" when fresh.
Additional citations: BOLIVIA: La Paz: Boeke 1452 (N). 
IIPPIA PRINGLEI Briq.

Additional bibliography: Lewis \& Elvin-Lewis, Med. Bot. 257 . 1977; Moldenke, Phytologia 39: 440--443 \& 454 (1978) and 40: 69 \& 81. 1978 .

Calzada refers to this species as a tree, $7 \mathrm{~m}$. tall, regular in growth, the "fruto en racimos color blancos alados", and found it growing in deciduous forests "in suelo cafe arcillozo, humedo", at $1675 \mathrm{~m}$. altitude, fruiting in February. Lewis \& Elvin-Lewis (1977) report that in Mexico the sap of this species is used to treat toothache.

Additional citations: MEXICO: Veracruz: Calzada 2170 (N).

LIPPIA PSEUDO-THEA (A. St.-Hil.) Schau.

Additional bibliography: Lewis \& Elvin-Lewis, Med. Bot. 391. 1977; Moldenke, Phytologia 39: 443--444. 1978.

Lewis \& Elvin-Lewis (1977) report the leaves of this plant used as a medicinal tea in Brazil.

LIPPIA ROSMARINIFOLIA Anderss.

Additional bibliography: Moldenke, Phytologia 39: 450- 452 . 1978.

Recent collectors describe this species as an erect shrub, I$4 \mathrm{~m}$. tall, sometimes tree-like, the branches loosely spreading, the "stems arch over and grow toward the ground and when lodged in a suitable crevice root to form a new plant; if the stem does not root directly it creeps prostrate along the rock surfaces". They have found it to be "common in [a] broad green strip running from [the] summit to [the] sea on well-vegetated aa lava with pumice in the crevices" and "common on scoria rim with much fine pumice deposited on the surface, partly grassy, partly with loose shrubby vegetation, at $300 \mathrm{~m}$. altitude, flowering in February, May, and June. The corollas are said to have been "pinkish to yellowish-pink" on Fosberg 45014, "pink" on Fosberg 45039, and "pale creamy-yellow or flesh-color" on Howell 9448.

Material has been misidentified and distributed in some herbaria as Nacraea laricifolia Hook. $f$.

Additional citations: GALAPAGOS ISLANDS: Albemarle: Howell 9448 $(W-2814443)$; Levêque 94 (W--2813702). Narborough: F. R. Fosberg $\underline{45014}(\mathrm{~W}-2828 \overline{124}), \underline{45039}(\mathrm{~W}-2828121)$ 。

IIPPIA ROSMARINIFOITA $f$. STEWARTI (Moldenke) Moldenke

Additional bibliography: Moldenke, Phytologia 39: 451 \& 452 . 1978.

Fosberg refers to this plant as a small shrub "common on rough aa lava with irregular vegetation, at $300 \mathrm{~m}$. altitude.

Additional citations: GALAPAGOS ISIANDS: Narborough: F. R. Fosberg 45067 (W-2828120). 
LIPPIA SALSA Griseb.

Additional bibliography: Ragonese \& Piccinini, Darwiniana 21: 53 \& 59. 1977; Moldenke, Phy tologia 39: 454--455. 1978.

Ragonese \& Piccinini (1977) record this species from San Juan, Argentina, with the vernacular names there of "yerba del cieroo" and "yerba del guanaco".

LIPPIA SALVIAEFOLIA Cham.

Additional bibliography: Moldenke, Phytologia 39: $455--456$. 1978.

Hatschbach refers to this plant as a shrub, 1.5 meters tall, and found it growing in sandy soil of cerrado along roadsides, flowering in April.

Additional citations: BRAZIL: Minas Gerais: Hatschbach $\underline{41313}$ (Ld).

\section{LIPPIA SCABERRIMA Sond.}

Additional bibliography: Lewis \& Elvin-Lewis, Med. Bot. 343. 1977; Moldenke, Phytologia 40: 58-59. 1978.

Lewis \& Elvin-Lewis (1977) report that the dried herbage of this species is considered hemostatic by native doctors in South Africa.

LIPPIA SCHLIMII Turcz.

Additional bibliography: López-Palacios, Fl. Venez. Verb. 418 , 419, 444-449, \& 651, fig. 104. 1977; Moldenke, Phytologia 40: 60--62 (1978) and 41: 135. 1978 .

Illustrations: Lopez-Palacios, Fl. Venez. Verb. [4 45 ], fig. 104.1977.

Lopez-Palacios (1977) cites the following collections from Venezuela: Lara: Steyermark 104919. Trujillo: Cuatrecasas, Ruiz-Terán, \& Lopez-Figueiras 28198; Ruiz-Terán \& Lbpez-Figueiras 2350; Ruiz-Terán \& Ibpez-Palacios 10414. He records the vernacular name, "saca candela".

LIPPIA SCHLIMII var. GLABRESCENS (Moldenke) Moldenke

Additional bibliography: L6pez-Palacios, Fl. Venez. Verb. 418 , 419, 446--449, \& 651, fig. 105. 1977; Moldenke, Phytologia 40: 61-62 (1978) and 41: 135. 1978.

Illustrations: Lopez-Palacios, F1. Venez. Verb. [447], fig. 105. 1977.

In his 1977 work Lopez-Palacios cites the following collections from Venezuela: Mérida: Gehriger 337; Linden 34l; Little 15310; Lopez-Palacios 1308, 2581; Matos 21; Ruiz-Terán 809, 1631, 2291; Ruiz-Terán \& López-Figueiras 8598; Ruiz-Terán, LópezFigueiras, \& Ibpez-Palacios 8246; Ruiz-Terán, López-Palacios, \& Rodriguez 6730; Tillett \& Honig 738-393; Trujillo 8170. Táchira: Badillo 3456 ; Bautista FAM.59; Madriz 79, 95; Ruiz-Terán \& LbpezFigueiras 1273; Steyermark 52271, 57469, 96983; Tillett 737-300; 
Tillett \& Honig 738-535. Trujillo: Ruiz-Terán \& Lobez-Figueiras 2247; Steyermark 97280. In a personal communication to me he lists the common names, "cancha", "gallinazo", "humo", "sacaojo", and "salvio".

Additional citations: COLOMBIA: Valle del Cauca: Cuatrecasas 2047 (W--2817824).

LIPPIA SCHOMBURGKIANA Schau.

Additional synonymy: Lippia schomburgkiana Moldenke, in herb.

Additional bibliography: Lopez-Palacios, FI. Venez. Verb. 418, 450-452, \& 651, fig. 106. 1977; Moldenke, Phytologia 40: 62-63. 1978.

IIlustrations: Iópez-Palacios, Fl. Venez. Verb. [451], fig. 106. 1977.

Lopez-Palacios (1977) comments that "Schauer....dice que es afín, pero distinta, a I. glandulosa Schauer, pero yo la considero mucho más cercana a I. origanoides H.B.K., de algunas de cuyas formas quizás sólo varia en rango infraespecifico, pues la única diferencia que se les observa es el olor." He cites from Venezuela the following collections: Bolivar: Connel \& Quelch 2; ImThurn 52; Irwin 402; Puiz-Terán \& Lopez-Palacios 11515.

LIPPIA TURBINATA Griseb.

Additional bibliography: Markgraf \& D'Antoni, Pollen Fl. Argent. 25, 98, 112, \& 115, pl. 42-362. 1978: Moldenke, Phytologia 40: 201 . 1978 .

Additional illustrations: Markgraf \& D'Antoni, Pollen Fl. Argent. pl. 42-362. 1978.

The pollen of this species is illustrated and described by Markgraf \& D'Antoni (1978).

\section{IIPPIA UMBEILATA Cav.}

Additional bibliography: Moldenke, Phytologia 40:68, 69, 73, \& 80-82. 1978 .

Jones encountered this plant at the edges of barrancas.

The Calzada 2170, distributed as I. umbellata, actually is I. pringlei Briq.

Additional citations: MEXICO: Morelos: G. N. Jones 23258 (Ld).

\section{ADDITIONAL NOTES ON THE GENUS VERBENA. XXVIII}

Harold N. Moldenke

VERBENA [Dorst.] I.

Additional synonymy: Verbena [Bauhin] L. ex Malag. Heras., 


\section{$2 \mathrm{BHL}$ Biodiversity Heritage Library}

Moldenke, Harold N. 1979. "Additional notes on the genus Lippia. XV." Phytologia 41, 145-151. https://doi.org/10.5962/bhl.part.20779.

View This Item Online: $\underline{\text { https://www.biodiversitylibrary.org/item/47696 }}$

DOI: https://doi.org/10.5962/bhl.part.20779

Permalink: https://www.biodiversitylibrary.org/partpdf/20779

\section{Holding Institution}

New York Botanical Garden, LuEsther T. Mertz Library

\section{Sponsored by}

The LuEsther T Mertz Library, the New York Botanical Garden

\section{Copyright \& Reuse}

Copyright Status: In copyright. Digitized with the permission of the rights holder.

Rights Holder: Phytologia

License: http://creativecommons.org/licenses/by-nc-sa/3.0/

Rights: https://biodiversitylibrary.org/permissions

This document was created from content at the Biodiversity Heritage Library, the world's largest open access digital library for biodiversity literature and archives. Visit BHL at https://www.biodiversitylibrary.org. 